\title{
A 55 Year-old Man With Mental Status Change and SEVERE ANEMIA
}

Sugeet Jagpal, MD, and Anastasia Shnitser, MD

\section{Case Presentation}

A 55 year-old male with past medical history significant for mental retardation, a stage IV sacral decubitous ulcer, iron deficiency anemia and gastrointestinal bleeding presented from a long term care facility for acute onset of respiratory distress and change in mental status. On presentation the patient was found to have a GCS of three, and he was emergently intubated in the emergency department for airway protection. Following intubation his vital signs were stable.

Upon review of his recent lab work, it was noted that patient had hemoglobin $(\mathrm{Hgb})$ of $6.4 \mathrm{~g} / \mathrm{dL}$ the day prior to admission with a known baseline of $8 \mathrm{~g} / \mathrm{dL}$. A blood transfusion was ordered. However, the transfusion was delayed secondary to difficulties in cross matching the patient's blood. His admission labs returned with a $\mathrm{Hgb}$ of $2.6 \mathrm{~g} / \mathrm{dL}$, a stool guiac test was negative, and an elevated LDH as well as positive direct Coombs C3 complement and IgG were noted. The patient was transfused two units of Oblood while the blood bank worked to determine which antibodies the patient had and find a suitable match. Eventually, the patient was diagnosed with cold agglutinin autoimmune hemolytic anemia based on an increase in his cold agglutin IgM antibody titer. At that time, the options were to either delay further transfusion as it would potentially worsen the hemolysis or to transfuse warmed blood.

Shortly after admission the patient became febrile, hypotensive and tachycardic. Despite treatment with antibiotics and fluids, the patient lost his pulse and required cardiopulmonary resuscitation. Lab work during the code showed a Hgb of $2.7 \mathrm{~g} /$ $\mathrm{dL}$, and the patient was given additional units of warmed blood. Unfortunately, the patient did not respond and expired within 24 hours of admission.

\section{Discussion}

Autoimmune hemolytic anemia (AIHA) is a disease in which antibodies bind to red blood cell surface antigens and initiate blood cell destruction. AIHA can be classified into warm and cold reactive antibody disease. With cold agglutinin AIHA, it is thought that the antibody binds to blood cells in the periphery (since blood cools as it passes through the smaller peripheral vessels), activating a complement cascade to the stage of $\mathrm{C} 3 \mathrm{~b}$. C3b continues to adhere to the red blood cells (RBCs) while they are in circulation. The C3bcoated RBCs encounter receptor specific macrophages, which then engulf the RBCs and are cleared in the liver.
Cold agglutinin AIHA can range in severity from mild to life threatening, and febrile illnesses can exacerbate chronic mild hemolysis due to the elevation in complements as part of the acute phase reaction. Diagnosis of this anemia is made by measuring the cold agglutin IgM antibody titers- the antibody titers measured at $4^{\circ} \mathrm{C}$ are greatly increased (up to $1: 1 \times 10^{6}$ ) from a normal low titer of $<1: 16$ at $4^{\circ} \mathrm{C}$. Also, the thermal amplitude of the antibody is increased, and the antibody binds to the surface of RBCs at temperatures as high as $28^{\circ}$ to $32^{\circ} \mathrm{C}$, when the normal thermal amplitude of the antibody does not allow binding between 20 to $37^{\circ} \mathrm{C} .^{1}$

Rapidly developing anemia with defects in tissue perfusion, such as in our patient, requires urgent treatment. However, treatment with blood transfusions can be difficult due to the ongoing hemolysis. Transfusion of warmed blood decreases the hemolysis because the antibody binding is impaired at warmer temperatures. Steroids are often given in conjunction with transfusions to blunt the patient's immune response. Inducing hypothermia is sometimes part of the treatment in attempt to maximize tissue oxygen delivery. ${ }^{2}$ Splenectomy, although mentioned in the literature, is usually not helpful since the macrophages are primarily cleared in the liver. There are also a few reports of successful treatment with monoclonal antibodies; however, they are not used for rapidly progressing hemolytic anemia. ${ }^{3}$

Patients with primary cold agglutinin disease usually have moderate anemia with occasional attacks of acrocyanosis when exposed to cold. These patients are generally elderly (70 to 80 years old) and have underlying malignancies. When a younger patient such as ours presents presents with acute onset of cold agglutinin disease, an infection with either Mycoplasma pneumoniae or infectious mononucleosis should be suspected. In these patients, additional treatment of the underlying disorder is helpful when anemia is not as rapidly progressive as in our patient.

\section{References}

1. Stein JH. Internal Medicine, Stein - 5th Ed (Chapter 89). Mosby Inc, 1998.

2. Campbell R, Marik PE. Severe Autoimmune Hemolytic Anemia Treated by Paralysis, Induced Hypothermia, and Splenic Embolization. Chest 2005; 127:678-681.

3. Berentsen S, Beiske K, Tjønnfjord GE. Primary chronic cold agglutinin disease: An update on pathogenesis, clinical features and therapy. Hematology 2007; 12: 361-370. 